\title{
Study on Prevention of Acute Radiodermatitis with Soft Silicone Film Dressing
}

\author{
MEI YING ZOU, DE JING XU, RONG ZHANG, YA XIN WANG, LING LI, JIANG TING HUANG AND XIANG YIN JI* \\ Department of Radiation Oncology, Jiangsu Cancer Hospital, The Affiliated Cancer Hospital of Nanjing Medical University, \\ Jiangsu Institute of Cancer Research, No. 42, Baiziting, Xuanwu District, Nanjing, Jiangsu 210009, China
}

\section{Zou et al.: Prevention of Acute Radiodermatitis with Soft Silicone Film Dressing}

\begin{abstract}
In order to compare the incidence and recovery effect of radiation dermatitis pain, to explore the effect of soft polysiloxane film dressing on radiation dermatitis. Patients with radiation dermatitis were randomly divided into the control group and the experimental group: the experimental group was treated with soft polysiloxane film dressing during the interval of radiotherapy, while the control group was treated with routine education and nursing. The cure rate of the two groups was compared and analyzed. The experimental results showed that the incidence of radiation dermatitis in the experimental group was lower than that in the control group, which proved that soft polysiloxane film dressing could prevent acute radiation dermatitis The curative effect of dermatitis is better.
\end{abstract}

Key words: Soft polysiloxane film, radiotherapy, dermatitis

Radiotherapy is an important method for the treatment of malignant tumors, but in the process of radiotherapy, it is easy to produce side effects, leading to the occurrence of radiodermatitis. Radiodermatitis refers to the direct damage of high-energy physical radiation to DNA molecules of human epidermal cells, which hinders the irreversibility of DNA, cell differentiation, cell mutation and basic damage of these tissues, resulting in dermatitis reaction and damage ${ }^{[1]}$. Scientific measures should be taken before and after radiotherapy to reduce the occurrence of radiation dermatitis ${ }^{[2]}$. The traditional treatment methods of radioactive dermatitis mostly include vitamin $\mathrm{C}$, hydrophilic gel, Vaseline, alcohol gauze and Aloe Vera complex vitamin E, so as to reduce the occurrence of radioactive dermatitis, reduce injury and prolong treatment time, but its practical application effect still needs to be improved ${ }^{[3]}$. Severe patients with acute radiation dermatitis are characterized by pruritus, peeling, blackening and blackening. Severe dermatitis is characterized by burning sensation, eczema, herpes, infection, suppuration and ulcer. Once acute radiation dermatitis occurs, it will greatly aggravate the pain of patients, prolong the length of hospital stay, and increase the ideological and economic burden of patients. Radiotherapy is necessary for most cancer patients ${ }^{[4]}$. In the process of radiotherapy, there will be different degrees of damage to the surrounding tissues. The most common is radiation dermatitis, and even cause local infection. Based on this, the research method of soft silicone film dressing to prevent acute radiation dermatitis was proposed ${ }^{[5]}$. From April 2019 to March 2020, soft silica gel dressing was used to prevent the occurrence of radiation-induced skin diseases.

\section{MATERIALS AND METHODS}

\section{Data collection:}

From April 2019 to March 2020, 100 patients with radiation dermatitis received radiotherapy. Patients with acute radiation dermatitis showed local congestion, edema, blisters, skin exudation, erosion and shedding ${ }^{[6]}$. Among them, 18 cases were caused by nasopharyngeal carcinoma, 23 cases by breast cancer, 2 cases by tongue cancer and 57 cases by others. There were 56 males and 44 females with an average age of 52.3 y (range, 19-65 y) ${ }^{[7]}$. There were 57 patients with acute radiodermatitis area less than $5 \mathrm{~cm}$ and 42 patients with acute radiodermatitis area more than $8 \mathrm{~cm}^{[8]}$.

KPS score of acute radiation dermatitis reaction degree: radiation dermatitis injury degree: Degree 0: no change; Degree 1: follicular, mild erythema, dry skin, less sweat; Degree 2: obvious erythema, macular 
wet dermatitis, moderate edema ${ }^{[9]}$; Degree 3: fusion, edema; Degree 4: necrosis, ulcer, bleeding ${ }^{[10]}$; During the course of the study, all malignant tumors received radiotherapy for the first time. All patients scored more than 70 on the KPS ${ }^{[11]}$.

\section{Experimental materials:}

Soft silicone film dressings, alcohol gauze, ordinary ultra-thin soft silicone dressings (made in Sweden), the use of the above materials can reduce the pain when changing dressings, dressings themselves can remain sticky, easy to take out and adjust ${ }^{[12]}$. It has sedative and anti-inflammatory effects.

\section{Research method:}

The patients were randomly divided into experimental group and control group. In the experimental group, soft polysiloxane film dressing was used to protect dermatitis (the dressing was taken off in the interval of radiotherapy, and bandaged in the interval of radiotherapy), and routine nursing was carried out at the same time ${ }^{[13]}$. The control group was only given routine nursing to improve the quality of nursing care. There was no significant difference in age, gender, radiation dose, KPS score between the two groups ${ }^{[14]}$.

$2 \mathrm{~d}$ before radiotherapy, on the basis of routine nursing, the patients were treated with soft silicone film dressing, soft silica gel, Vaseline, MEBO and other materials to irradiate the burn wound until the end of radiotherapy ${ }^{[15]}$. Nurses are responsible for the daily care and medication of patients. The data collector does not directly participate in the management of patients, but only observes the patient's condition, fills in the evaluation form, guides the patient to fill in the symptom evaluation form, closely observes the patient's condition and fills in the evaluation form at the designated time point ${ }^{[16]}$. If there are abnormal conditions or problems, contact the doctor and medical staff in time.

After the soft polysiloxane film dressing was cut according to the size of radiation field $(10 \times 10 \mathrm{~cm} / 15$ $\times 15 \mathrm{~cm}$ ), the dressing pasted on the bend of radiotherapy area was cut into small incision to improve the contact stability between the dressing and radiation field ${ }^{[17]}$. Use a $2 \mathrm{~cm}$ wide white scarf to tie the neck tightly for $1-2 \mathrm{w}$ and fix the bandage slightly. At the same time, the white belts on the left and right sides should be fixed with adhesive tape to prevent movement. It was removed during radiotherapy and chemotherapy and applied after radiotherapy and chemotherapy ${ }^{[18]}$. Replace every
3-5 d. In case of water or sweat, it should be replaced in time.

Before the end of Vaseline medication, once a day in the morning and evening, $30 \mathrm{~min}$ each time. In the control group, alcohol gauze, vaseline and other materials were used as dressing on the burn wound until the end of radiotherapy. Nurses are responsible for the daily care and medication of patients ${ }^{[19]}$. Wipe gauze $1 \mathrm{~h}$ before radiotherapy to avoid residual.

Radiotherapy combined with single wound treatment and mixed suspension agent were used for nursing care $^{[20]}$. Normal saline cotton ball was used to clean the wound and the surrounding dermatitis, and the necrotic and exfoliated epidermis was cleaned. Sterile dressing was used to dry the wound and the surrounding dermatitis area. According to the size of the wound in the dermatitis area, a new soft silicone dressing was applied to the wound and the surrounding dermatitis area. The wet exudation of the dressing surface was observed, and the dressing was replaced every 2-7 d. The size (thickness) of soft silicone dressing should be selected according to different incision, and the incision size should be larger than $1 \mathrm{~cm}$. When using, the incision should be smooth to avoid bubbles on the contact surface. The dressing change interval was determined according to the wound exudation, and the dressing was changed in time after the wound was wet. After 24-48 h, there was no leakage on the surface of the dressing, the granulation tissue was fresh and the color was bright red. During this period, the soft silicone film dressing was replaced every 3-4 $\mathrm{d}$ in the experimental group.

\section{Evaluating indicator:}

The incidence of radiation dermatitis and pain level after radiation was used as evaluation indexes. Based on the United States radiotherapy classification standard, the evaluation rate of radiation dermatitis was: Grade 0: no change; Grade 1: follicular dark erythema, or dry off, or hair removal, or sweating reduction; Grade 2: flaky erosion/moderate erythema; Grade 3: erosion outside the fold, punctate edema; Grade 4: ulcer, bleeding, necrosis.

The evaluation indexes of pain level after radiation were as follows: Grade 0 showed no pain, Grade 1-3 showed mild pain, grade 4-6 showed moderate pain, and grade 7-10 showed severe pain. Evaluation time was after $12 \mathrm{~h}$ of treatment, the curative effect is significant, $2 \sim 3 \mathrm{~d}$ after healing. The rehabilitation evaluation criteria were as follows: Complete cure: local dermatitis 
dry smooth, no scab, itching symptoms disappear, does not affect the curative effect. The effect is very good: after treatment, the wound gradually shrinks and heals in 7-12 d. Even if the radiotherapy is interrupted, the treatment can still be completed.

Good effect-Local dermatitis dry, pain and itching symptoms disappear, scab.

Poor effect-15 d later, the symptoms did not improve significantly, the local exudation of purulent secretions increased, and other treatment methods were needed to complete the radiotherapy

\section{Recovery evaluation algorithm of radiation der- matitis:}

In order to better protect the research results, we optimize the recovery evaluation algorithm of radiation dermatitis, and collect and analyze the local skin image information based on the regional features of patient's dermatitis. Because the obtained regional features of skin inflammation are rectangular features, and the average value of the three channels in the rectangular feature $\bar{X}_{\mathrm{k}}$, variance $\mathrm{X}_{\mathrm{ij}}$, slope $\mathrm{S}_{\mathrm{k}}$. In general, there is a big difference in the area values, so we should first standardize the image features of the patient area, and the standardization formula is as follows: $\bar{X}_{\mathrm{ik}}=\mathrm{X}_{\mathrm{ik}} \overline{\mathrm{X}}_{\mathrm{k}} /$ $S_{k}$ (1). Based on the above algorithm, the function of dimension can be eliminated. The enhanced feature vector is used to fuse the skin defect features of three dermatitis regions. Let the skin image sample space of dermatitis region be $\Omega$, the texture feature space be $\mathrm{x}$, $\mathrm{y}, \mathrm{Z}$, and the feature vector of dermatitis sample in the feature space of database be $\theta$ then: $\theta=\bar{X}_{\mathrm{ik}}(\alpha \mathrm{x}, \beta \mathrm{y}, \gamma \mathrm{z})(2)$

Where, $\alpha, \beta, \gamma$ represents spatial features respectively, and $\alpha+\beta+\gamma \geq 1$. Its value is determined by the severity of the patient's dermatitis. Based on this, the information collection method of the patient's dermatitis area is further optimized to ensure the recognition effect. The principle of the information collection and recognition of the patient's dermatitis area as shown in fig. 1.

As shown in fig. 1, using linear transformation to convert the low-dimensional space to the high-dimensional space can easily find the feature plane image information in the high-dimensional space. Due to the use of training set and kernel function for description, in order to ensure the accuracy of classification and the overall accuracy of the algorithm, the evaluation indicators for dermatitis patients are further optimized. The specific algorithm is: $\mathrm{ACC}_{\mathrm{i}}=\mathrm{TP}_{\mathrm{i}} /$ num $_{\mathrm{i}}+\theta$ (3), $\mathrm{ACC}=\mathrm{TP}_{0}+\mathrm{TP}_{1}+\mathrm{TP}_{2}+\mathrm{TP}_{3}+\mathrm{TP}_{4}+\mathrm{TP}_{5} / \sum$ num $_{\mathrm{i}}(4)$

Where TP is the number of samples correctly classified, $\mathrm{um}_{\mathrm{i}}$ is the total number of test samples of $\mathrm{N}$ categories. In the biomedical field, the detection and classification of skin lesions mainly adopt fully convolutional network (FCN) structure and cnofen structure. Based on this, the classification is carried out at the pixel level, the image segmentation is realized at the semantic level, the image is classified from the skin image level, and the overall category of the image is analyzed. Based on this, the convolution neural network is further used to detect the dermatitis image, and the specific method is shown in the following fig. 2. According to the above steps, input $100 \times 100 \mathrm{~mm}$ dermatitis image,

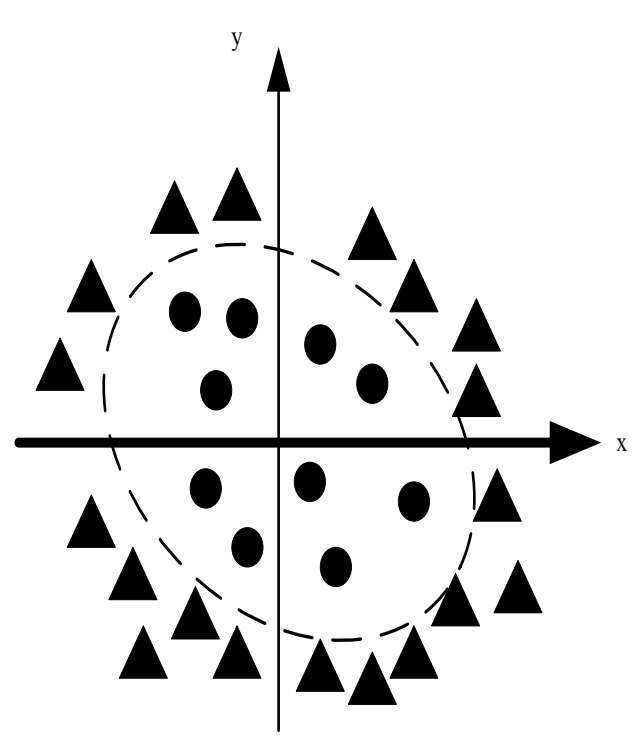

(a) Low dimensional space

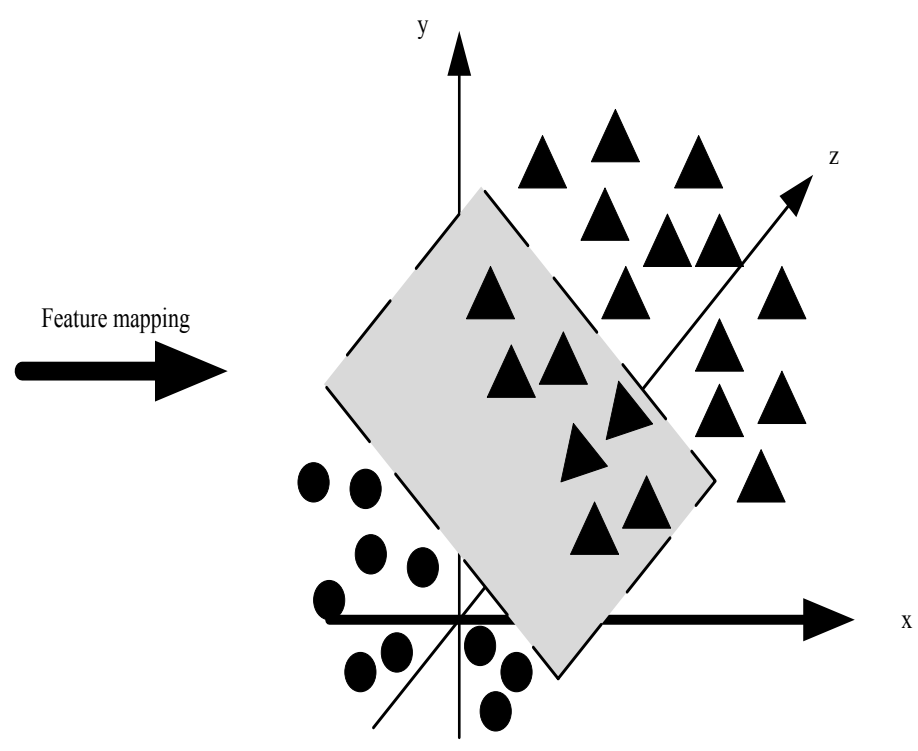

(b) High dimensional space

Fig. 1: Principle of information collection and recognition of patients' dermatitis area 
convolute through convolution layer and filter layer, and activate convolution with ReLU function to avoid gradient disappearance, keep the convergence rate of the model stable, and then reduce the eigenvalue through pooling layer. After a series of convolution, nonlinear and pooling operations, features are sent to the whole connection layer. So as to detect the degree of dermatitis,. Ensure the accuracy of the research results.

\section{RESULTS AND DISCUSSION}

The experimental platform of feature skin defect detection method is $17-7700 \mathrm{KCPU} @ 4.20 \mathrm{GHz}$ It is based on windows 10 ultimate 10, combined with visual studio 2013 system. The integrated memory of openco 2017 processor is $128 \mathrm{~GB}$. The data were analyzed by SPSS 13.0 software. Before the formal experiment, 10 patients were selected for the pre experiment to discover and solve the possible problems in the research process in time, and modify the specific experimental process of the research process. The expected experimental results are only used to improve the study design. Two tests were used in both groups. If the research result value $p<0.05$, the incidence of radiation dermatitis was significant. The study found that there was a significant difference in the incidence of injury between the two groups, the specific test results are shown in Table 1.

To further compare the possibility of occurrence of hypodermatitis under different treatments, the specific results are shown in Table 2. In the course of the study, it was found that there was a significant difference in the incidence of pain between the experimental group and the control group $(\mathrm{p}<0.01)$, the comparison results of the skin pain of the two groups of patients are shown in Table 3. Compare the investigations of patients with dermatitis and pain under different methods, as shown in Table 4. The number of dressing changes, healing time, and the incidence of local side effects of the two groups of patients were observed. The results are shown in Table 5. Further comparative analysis of the degree of mesotheliitis in the two groups of patients was recorded, as shown in Table 6. To further compare

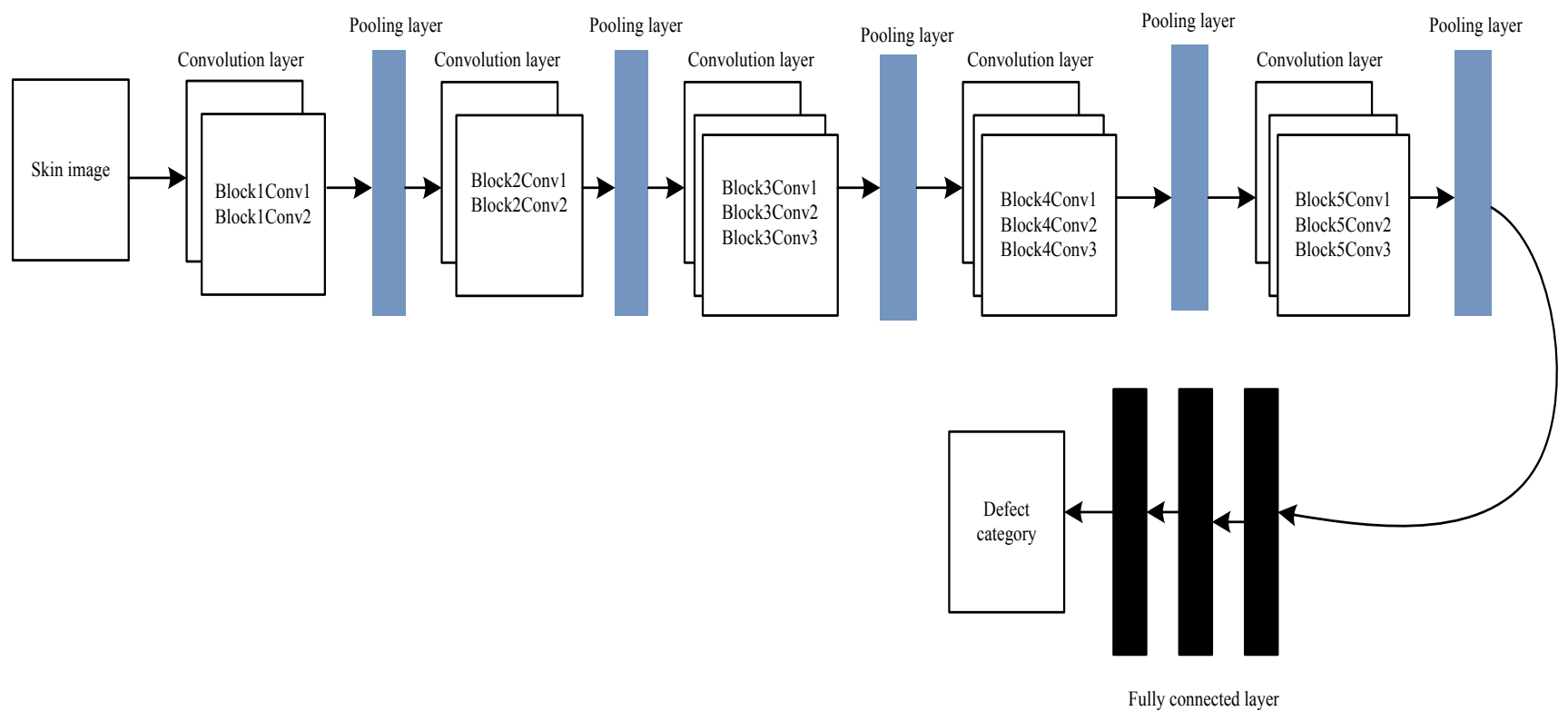

Fig. 2: Identification and judgment process of radio dermatitis

TABLE 1: COMPARISON OF THE INCIDENCE OF RADIATION DERMATITIS BETWEEN THE TWO GROUPS (\%)

\begin{tabular}{lccccccc}
\hline Group & Number of cases & Normal & Level 1 & Level 2 & Level 3 & Grade 4 & Incidence rate \\
\hline Experiment group & 46 & $22(47.8)$ & $16(34.8)$ & $7(15.2)$ & $1(2.2)$ & $0(0)$ & $24(52.2)$ \\
Control group & 50 & $6(12.0)$ & $14(28.0)$ & $21(42.0)$ & $9(18.0)$ & $0(0.0)$ & $44(88.0)$ \\
\hline
\end{tabular}

TABLE 2: INCIDENCE RATE OF DERMATITIS UNDER DIFFERENT TREATMENT METHODS

\begin{tabular}{lccc}
\hline Group & Number of cases & Age & Incision length \\
\hline Soft polysiloxane & 40 & $30.2 \pm 6.5$ & $3.56 \pm 0.87$ \\
Dry gauze & 40 & $30.8 \pm 6.7$ & $3.87 \pm 0.46$ \\
Vaseline & 40 & $28.9 \pm 7.3$ & $3.10 \pm 1.22$ \\
Alcohol yarn & 40 & $30.2 \pm 6.2$ & $3.79 \pm 0.62$ \\
\hline
\end{tabular}


and analyze the degree of mesodermatitis scar between the two groups of patients to record, as shown in Table 7.

In the experiment, 14 cases with incision diameter less than $5 \mathrm{~cm}$ were fixed with soft silicone film dressing and healed $3 \mathrm{~d}$ later. The area of dermatitis was larger than $5 \mathrm{~cm} \times 5 \mathrm{~cm}$ in 12 cases. The wound healed $5 \mathrm{~d}$ after the dermatitis area was fixed with soft silicone film dressing, and radiotherapy was continued. Before the end of radiotherapy, 12 cases were grade I and II dermatitis, 34 cases were grade III dermatitis; 54 cases were $42.7 \%$, including 25 cases in soft silicone film dressing group, 25 cases in vaseline group, 25 cases in alcohol gauze group and 25 cases in dry gauze group. Specifically as shown in Table 8. The comparative analysis of patients RISRAS scores are shown in fig. 3 .

According to the interaction curve of different treatment regimens, the interaction can be preliminarily judged,

TABLE 3: COMPARISON OF SKIN PAIN BETWEEN THE TWO GROUPS (\%)

\begin{tabular}{lcccccc}
\hline Group & Number of cases & No pain & Mild pain & Moderate pain & Severe pain & Incidence of pain \\
\hline Experiment group & 46 & $27(58.7)$ & $14(30.4)$ & $5(10.9)$ & $0(13.9)$ & $19(41.3)$ \\
Control group & 50 & $5(10.0)$ & $16(32.0)$ & $18(36.0)$ & $11(22.0)$ & $45(90.0)$ \\
\hline
\end{tabular}

TABLE 4: INVESTIGATION RESULTS OF DERMATITIS AND PAIN IN PATIENTS WITH DIFFERENT METHODS

\begin{tabular}{|c|c|c|c|c|c|c|c|}
\hline \multirow{2}{*}{ Group } & & \multirow{2}{*}{$\begin{array}{c}\text { Number of } \\
\text { cases }\end{array}$} & \multicolumn{5}{|c|}{ Grade of incision pain (cases) } \\
\hline & & & Grade 0 & Level 1 & Level 2 & Level 3 & Level 4 \\
\hline \multirow{3}{*}{$\begin{array}{l}\text { Soft } \\
\text { polysiloxane }\end{array}$} & $0-48 \mathrm{~h}$ after radiotherapy & 40 & 12 & 22 & 6 & 0 & 0 \\
\hline & 48-96 $\mathrm{h}$ after radiotherapy & 40 & 28 & 12 & 0 & 0 & 0 \\
\hline & 96-144 h after radiotherapy & 40 & 40 & 0 & 0 & 0 & 0 \\
\hline \multirow{4}{*}{ Dry gauze } & 0-48 h after radiotherapy & 40 & 4 & 11 & 16 & 5 & 4 \\
\hline & 48-96 $\mathrm{h}$ after radiotherapy & 40 & 10 & 21 & 5 & 4 & 0 \\
\hline & 96-144 h after radiotherapy & 40 & 24 & 10 & 6 & 0 & 0 \\
\hline & 0-48 h after radiotherapy & 40 & 4 & 14 & 18 & 4 & 0 \\
\hline & 48-96 $\mathrm{h}$ after radiotherapy & 40 & 15 & 11 & 13 & 0 & 0 \\
\hline \multirow[t]{2}{*}{ Vaseline } & $96-144 \mathrm{~h}$ after radiotherapy & 40 & 25 & 12 & 3 & 0 & 0 \\
\hline & 0-48 h after radiotherapy & 40 & 0 & 13 & 15 & 8 & 4 \\
\hline \multirow[t]{2}{*}{ Alcohol yarn } & $48-96 \mathrm{~h}$ after radiotherapy & 40 & 3 & 13 & 14 & 9 & 1 \\
\hline & $96-144 \mathrm{~h}$ after radiotherapy & 40 & 10 & 14 & 13 & 3 & 0 \\
\hline
\end{tabular}

TABLE 5: TIMES OF DRESSING CHANGE, HEALING TIME AND LOCAL SIDE EFFECTS OF PATIENTS

\begin{tabular}{lcccc}
\hline Group & Number of cases & Dressing change times (Times) & Healing time (d) & $\begin{array}{c}\text { Local skin allergy } \\
\text { (case report) }\end{array}$ \\
\hline Soft polysiloxane & 40 & $3.95 \pm 1.78$ & $6.46 \pm 2.38$ & 0 \\
Dry gauze & 40 & $5.48 \pm 3.75$ & $8.34 \pm 3.40$ & 3 \\
Vaseline & 40 & $5.40 \pm 3.79$ & $8.24 \pm 3.27$ & 0 \\
Alcohol yarn & 40 & $5.48 \pm 3.65$ & $8.26 \pm 3.40$ & 6 \\
\hline
\end{tabular}

TABLE 6: THE DEGREE OF REGIONAL DERMATITIS AMONG GROUPS

\begin{tabular}{lccccc}
\hline \multirow{2}{*}{ Group } & \multirow{2}{*}{ Number of cases } & \multirow{2}{*}{ No scab } & \multicolumn{3}{c}{ Scab (cases) } \\
\cline { 4 - 6 } & & & Light & Moderate & Severe \\
\hline Soft polysiloxane & 40 & 33 & 5 & 2 & 0 \\
Dry gauze & 40 & 0 & 22 & 12 & 6 \\
Vaseline & 40 & 15 & 15 & 10 & 0 \\
Alcohol yarn & 40 & 2 & 20 & 13 & 5 \\
\hline
\end{tabular}

TABLE 7: THE DEGREE OF DERMATITIS SCAR BETWEEN THE TWO GROUPS

\begin{tabular}{lccccc}
\hline Group & Number of cases & Invalid (\%) & Effective rate (\%) & Significant effect (\%) & Recovery rate (\%) \\
\hline Soft polysiloxane & 35 & $0(0)$ & $3(8.6)$ & $8(22.9)$ & $24(68.6)$ \\
Dry gauze & 35 & $7(20.0)$ & $10(28.6)$ & $10(28.6)$ & $8(22.9)$ \\
Vaseline & 36 & $4(11.1)$ & $8(22.2)$ & $11(30.6)$ & $13(36.1)$ \\
Alcohol yarn & 38 & $8(21.1)$ & $10(26.3)$ & $11(28.9)$ & $9(23.7)$ \\
\hline
\end{tabular}


TABLE 8: PATHOLOGICAL DIFFERENCES OF PATIENTS

\begin{tabular}{lcccc}
\hline & Squamous cell carcinoma & Non keratinocyte carcinoma & Differentiated carcinoma & Total \\
\hline Soft polysiloxane & 11 & 7 & 8 & 26 \\
Dry gauze & 10 & 6 & 9 & 25 \\
Vaseline & 11 & 1 & 12 & 24 \\
Alcohol yarn & 32 & 14 & 29 & 75 \\
\hline
\end{tabular}

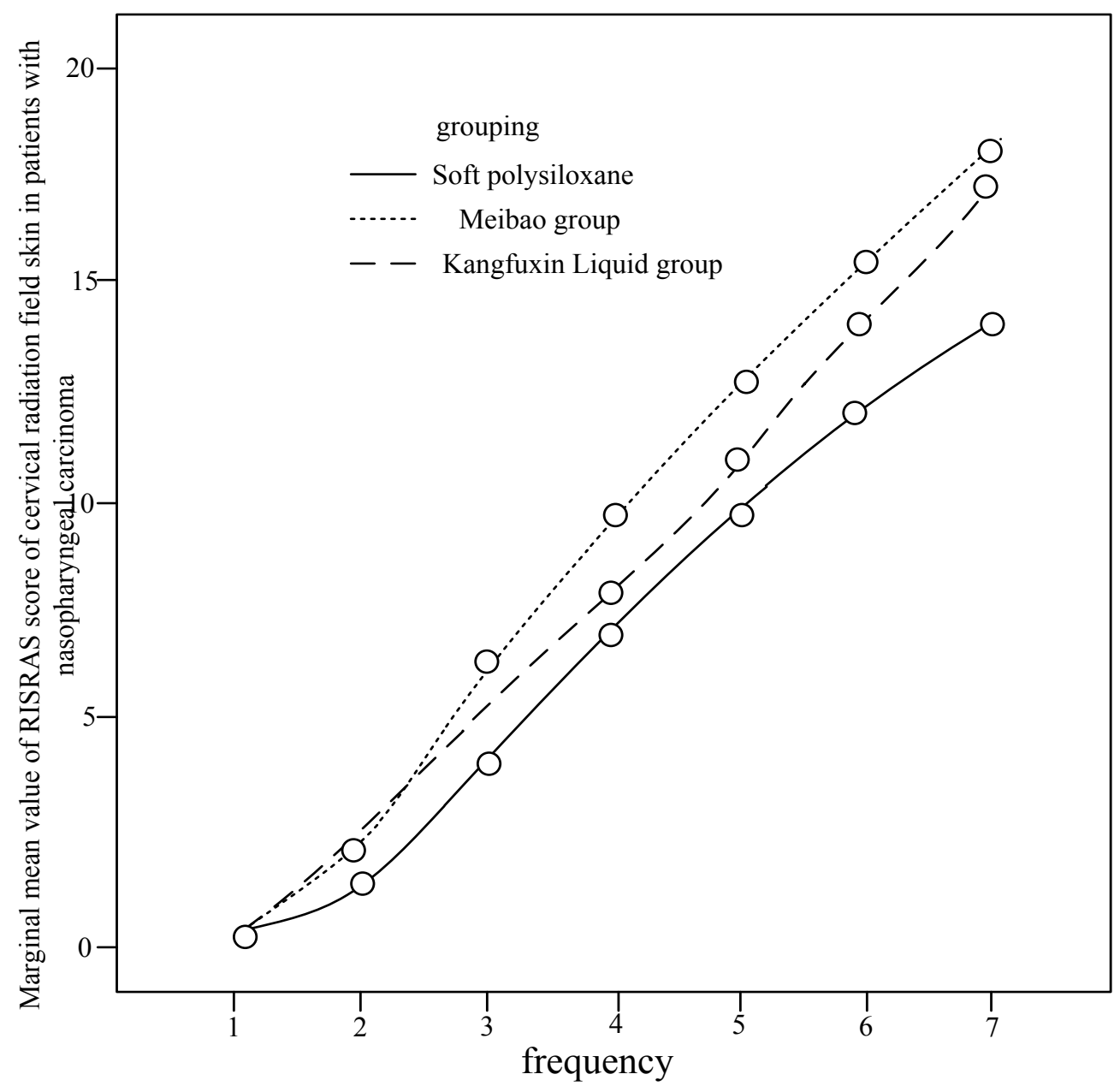

Fig. 3: Comparative Analysis of patients' RISRAS score, grouping, (_) soft polysiloxane, (......) meibao group, (-------) kangfuxin liquid group

and the change trend of RISRAS score in patients with acute radiation dermatitis can be directly observed. The change trend of RISRAS score of acute radiation dermatitis was consistent, and it increased with the extension of time. Therefore, it can be considered that the RISRAS score of patients increases with the increase of the total dose of radiotherapy.

The occurrence of radiation dermatitis is related to radiation quantity, radiation type, radiation point and external friction. It has certain latency and obvious phase characteristics, and has its own characteristics. After the injury, even if the radiotherapy stopped, the wound would continue to deteriorate; the wound healing was slow and lasted for a long time. In the past, the treatment of radiation dermatitis mainly used exposure drying method, external application of scald ointment, rifampicin, vaseline and other external application, resulting in long-term clinical application effect is not obvious. Dermatitis wound healing is a new concept that has been studied most recently. The use of soft silicone film dressing can reduce the frequency of dressing change, reduce the workload of medical staff, promote wound healing and reduce the cost. If the frequency of dressing change reaches 3-7 d, the soft silicone film dressing can be changed in one day at most. The soft silicone film dressing is made up of a soft silicone wound contact layer, an elastic polyurethane foam absorbing layer and an outer three layer coating. It has the functions of penetration, waterproof, suction, adhesion and suction. Soft silicone film dressing is a 
kind of high molecular organic compound, which has the functions of absorbing leakage, keeping wound moist, healing environment and promoting debridement. Soft polysiloxane film dressing antibacterial waterproof film can be gently attached to the dermatitis around the wound, but not attached to the wound, reduce the damage of new granulation tissue, reduce bleeding and pain, and will not lead to skin shedding and pain. After adsorbing a large amount of soft mud by soft polysiloxane film dressing, it can expand at the same time, prolong the dressing time, reduce the frequency of dressing change, simplify the operation, significantly reduce the nursing workload, reduce the physiological pain, psychological pressure and economic burden of patients, improve the quality of life, shorten the course of treatment, which is worthy of clinical promotion.

Our results have showed that the quality of radiation dermatitis can be better protected by the three layer soft silicone film dressing, which is mainly composed of wound contact layer, elastic polyurethane foam absorbing layer and breathable waterproof outer membrane. It can be better absorbed in a dry environment, which is conducive to wound healing. Soft polysiloxane film dressing can gently adhere to the skin around the wound, can effectively protect the post wound dermatitis, will not adhere to the wound and has good compliance. When the nurses take out the soft polysiloxane film dressing, it will not cause skin shedding and pain, and the pain will be significantly reduced after use. Therefore, it is concluded that soft polysiloxane can effectively prevent the development of dermatitis radiotherapy, improve the compliance of patients, and improve the quality of life, which is worthy of clinical application.

\section{Acknowledgements:}

This work was supported by Scientific Research Project of Jiangsu Cancer Hospital (NO.ZH201503)

\section{Conflicts of interest:}

The authors report no conflicts of interest.

\section{REFERENCES}

1. Pang B, Zhang J, Pang M, Zhao P, Yang Z, Feng S, et al. Design and preparation of a new polyuria-polysiloxane-polyether copolymer with a block soft segment prepared by utilizing azaMichael addition reaction. Polymer Chem 2018;9(7):869-77.

2. Yang N, Gao M, Li J, Lu K. Nickel containing magnetoceramics from water vapor assisted pyrolysis of polysiloxane and nickel 2, 4-pentanedionate. J Am Ceramic Soc 2020;103(1):145-57.

3. Bharwal AK, Manceriu L, Alloin F, Iojoiu C, Dewalque J,
Toupance $\mathrm{T}$, et al. Bimodal titanium oxide photoelectrodes with tuned porosity for improved light harvesting and polysiloxane-based polymer electrolyte infiltration. Solar Energy 2019;178:98-107.

4. Dastjerdi R, Rouhollahi F, Beigi MH, Nasr-Esfahani MH. Mechanism of formation of soft and elastic nanoflowers; a key major guideline. J Cleaner Product 2018;200:331-41.

5. Opris DM. Polar elastomers as novel materials for electromechanical actuator applications. Adv Mater 2018;30(5):1703678.

6. Sezen D, Bolukbasi Y, Cebi CC, Selek U. Abstract P3-12-18: Comparison of three different topical agents on prevention of acute radiodermatitis during breast cancer radiotherapy. Cancer Res 2019;79(4):3-18.

7. Rzepecki A, Birnbaum M, Ohri N, Daily J, Fox J, Bodner W, et al. Characterizing the effects of radiation dermatitis on quality of life: a prospective survey-based study. J Am Academy Dermatol 2019:S0190-9622.

8. Wasiak J, Tyack Z, Tacey M, Young A, Shen A, Jnr CF. Poor Methodological Quality but Higher Reporting Standards Seen in Systematic Reviews in Radiation Dermatitis. Int J Radiation Oncol Biol Physics 2019;105(1):E462.

9. Kim S, Kim J, Hwang M, Kim M, Jo SJ, Je M, et al. Smartphonebased multispectral imaging and machine-learning based analysis for discrimination between seborrheic dermatitis and psoriasis on the scalp. Biomed Optic Exp 2019;10(2):879-91.

10. Byers RA, Maiti R, Danby SG, Pang EJ, Mitchell B, Carré MJ, et al. Sub-clinical assessment of atopic dermatitis severity using angiographic optical coherence tomography. Biomed Optic Exp 2018;9(4):2001-17.

11. Bogen KT, Garry MR. Risks of Allergic Contact Dermatitis Elicited by Nickel, Chromium, and Organic Sensitizers: Quantitative Models Based on Clinical Patch Test Data. Risk Anal 2018;38(5):1036-51.

12. Whittington CM, Lo WY. Prevention of nickel release from electroplated articles in the context of allergic contact dermatitis: further outcomes. Transactions of the IMF 2018;96(2):63-70.

13. Vishalakshi GJ, NaveenKumar SK, Hemshekhar M, Mahendra M, Kemparaju K, Girish KS. Para tertiary butyl catechol (PTBC), an industrial antioxidant induces human platelet apoptosis. Environment Toxicol 2019;34(3):262-70.

14. Palma G, Conson M, Xu T, Hahn SM, Durante M, Mohan $\mathrm{R}$, et al. Severe Radiation Induced Dermatitis after IMRT or Proton Therapy for Thoracic Cancer Patients. Int J Radiation Oncol Biol Physics 2019;105(1):S6. Liu X, Michael S, Bharti K, Ferrer M, Song MJ. A biofabricated vascularized skin model of atopic dermatitis for preclinical studies. Biofabrication 2020;12(3):035002.

15. Yang G, Lee HE, Shin SW, Um SH, Lee JD, Kim KB, et al. Efficient transdermal delivery of DNA nanostructures alleviates atopic dermatitis symptoms in nc/nga mice. Adv Functional Material 2018;28(40):1801918.

16. Shah KH, Oza MJ. Comprehensive Review of Bioactive and Molecular Aspects of Moringa Oleifera Lam. Food Rev Int 2020;3:1-34.

17. Kim YM, Oh I, Kim J, Kang YH, Ahn K. Harmful Effects of Ambient Nitrogen Dioxide on Atopic Dermatitis: Comparison of Exposure Assessment Based on Monitored Concentrations and Modeled Estimates. Atmosphere 2020;11(9):921.

18. Jiang X, Wang W, Huang X, Song A, Lu L, Lu X, et al. 
Evaluate the Effect of Inhibiting Pathogenic Bacteria and Fungus of Eczematous Dermatitis and Antioxidant Activity of Phenolic from Qinglicao (Polygonum chinense L. Var. chinense) Biotechnol 2018;17(2):62-68.

19. Aoki H, Chuma S, Iba Y, Tashiro H, Watanabe N, Oyama H. Comparison of Bioactive Components in Tempeh Produced by Three Different Rhizopus Starters and Immunomodulatory Effect of Tempeh on Atopic Dermatitis Mice. Food Sci Technol Res 2020;26(5):665-72.
This is an open access article distributed under the terms of the Creative Commons Attribution-NonCommercial-ShareAlike 3.0 License, which allows others to remix, tweak, and build upon the work non-commercially, as long as the author is credited and the new creations are licensed under the identical terms

This article was originally published in a special issue,

"Evolutionary Strategies in Biomedical Research and

Pharmaceutical Sciences" Indian J Pharm Sci 2020:83(3)

Spl issue;49-56 